\title{
INTERFEROMETRY AND LUNAR OCCULTATIONS: STATUS AND PROSPECTS OF GROUND-BASED MILLIARCSECOND RESOLUTION OBSERVATIONS
}

\author{
A. Richichi ${ }^{1}$ \\ RESUMEN
}

Las ocultaciones lunares y la interferometría de gran base son dos métodos directos de obsevación capaces de lograr resoluciones de milisegundos de arco para estrellas binarias. Examinamos el estado actual de estas dos técnicas y, en particular, sus ventajas y desventajas, sus limitaciones y su accesibilidad para programas de investigación sobre binarias. También examinamos las perspectivas a corto plazo en relación a la disponibilidad de instrumentos interferométricos grandes como el VLTI.

\section{ABSTRACT}

Lunar occultations and long-baseline interferometry are two direct observational methods capable of delivering milliarcsecond resolution of binary stars. We will review the status of these two techniques, particularly with reference to their respective advantages, limitations, and accessibility for binary star research programs. We will also illustrate the near-term prospects, in connection with the availability of large modern interferometric facilities such as the VLTI.

\section{Key Words: BINARIES: GENERAL - OCCULTATIONS - TECHNIQUES: INTERFEROMETRIC}

\section{INTRODUCTION}

In the study of binary and multiple stars, as well as with many observational areas in astronomy, high angular resolution is of fundamental importance. In normal imaging observations, angular resolution is limited by the size of the telescope and, at least from the ground, by turbulence in the atmosphere. These limitations can be only partially overcome by building larger telescopes and by placing them in favorable sites, and ultimately in space. The seeing at the best sites is of order 0.5 arcseconds, and the diffraction limit of a $10-\mathrm{m}$ class telescope is of order 0.02 arcseconds in the near-IR (the best working range for the adaptive optics machinery needed to correct atmospheric degradations). However, investigations of binary stars often demand angular resolutions at the milliarcsecond (mas) level and beyond: this is required among others for accurate orbital motions, for the detection of close companions, and for the determination of stellar masses. It is then clear that means must be used, other than single mirror telescopes.

Lunar occultations (LO) and long-baseline interferometry (LBI) fall into the category of specialized techniques which can obtain this kind of performance. It is interesting to note that in both cases the first suggestions and experiments took place at the turn of the last century, only to be abandoned

\footnotetext{
${ }^{1}$ European Southern Observatory
}

in view of challenging technical and theoretical difficulties in their application. The parallel between the two techniques continued later. Lunar occultations were resurrected in the 1950's, although it took another two decades before they could finally establish themselves as one of the leading high-angular resolution methods, thanks to the introduction of IR detectors. Interferometry was rediscovered in the 1970's, and also in this case the key to its success was the application in the near-IR. The infrared range offers several advantages for both techniques: in the case of LO, the amount of scattered light from the Moon is much lower and therefore the sensitivity is highly improved; in the case of LBI, the requirements on atmospheric, optical and mechanical disturbances are much relaxed.

\section{OVERVIEW}

In spite of these interesting common aspects in their development, LO and LBI are completely different in their operating principles. LO consist of recording the diffraction pattern produced as the Moon moves over a background source. Since the phenomenon occurs in space, it is relatively insensitive to atmospheric turbulence. Here, the "telescope" is represented by the Moon's limb and therefore the "resolution" is independent of our terrestrial apparatus, to a first approximation. The highangular resolution information is embedded in the 


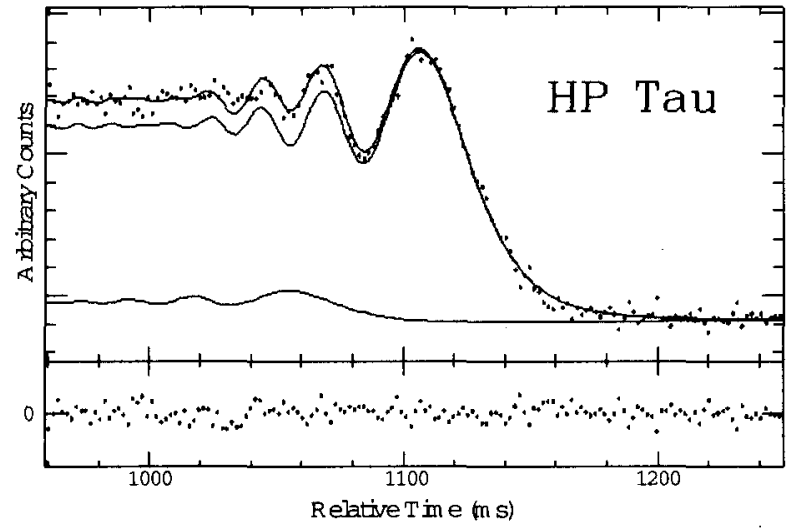

Fig. 1. Example of a lunar occultation light curve of a close binary star, HP Tau (adapted from Richichi et al. 1994). The upper panel shows the measured intensity points as dots, while the solid lines from bottom up are the model for the companion, the model for the primary and the total fitted model. In the lower panel, the fit residuals are shown.

fringe pattern, and it can be retrieved by a number of methods, either based on a model of the occulted source or model-independent. The phenomenon is chromatic, and typical temporal scales require sampling rates of order $1 \mathrm{~ms}$ in the near-IR (see Fig. 1).

LBI uses the combination of two or more telescopes at a large distance to achieve the angular resolution equivalent to their separation. As shown schematically in Fig. 2, interference fringes are produced when the wavefronts produced by two telescopes are combined exactly in phase. The contrast of the fringes as a function of baseline, the so-called visibility, is directly related to the size of the source. In general, the results of LBI are better interpreted in the Fourier domain. The problem of inverting this information to retrieve images suffers, at least in the optical/IR domain, from the fact that the sampling of the Fourier space is usually quite limited (i.e., only a few baselines are practically observed) and that the phase information is lost. Therefore, it is easiest to study simple objects such as stellar disks and binary systems, for which parametrized models can be provided and easily fitted to the interferometric measurements.

In the case of a simple binary system with two unresolved components, the visibility curve has a cosine shape as shown in Fig. 2, where the frequency of the cosine is related to the separation and the contrast to the brightness ratio. In this case, few measurements (ideally only two) are sufficient to determine these parameters. In the general case in which both sources are resolved, the visibility has a more complicated shape and a more extensive coverage of baselines is needed to derive all parameters. It is noteworthy that significant advances are being made to overcome the limitations of model fitting and produce actual images, for example by the socalled closure phases (see Hummel 2004).

\section{SOME STATISTICS}

It is interesting to evaluate the relative contribution of the two high angular resolution techniques considered here in the context of general observational results on binary stars. The CHARM catalogue (Richichi \& Percheron 2002) is a valuable source of information, collecting a large number of LO and LBI measurements. Of the 3248 entries in CHARM, just over $10 \%$ refer to binary or multiple systems. It is important to note that each entry represents an independent measurement, and not necessarily a different source.

Notwithstanding a few cases of indirect estimates, 31 entries are from LBI and 334 from LO measurements. For both LBI and LO entries, about half are in the visible and half in the infrared range. One should note that this does not include the large sample of LO events (about 7000) observed by Evans et al. (1986), and references therein. This interesting database is unfortunately not available electronically. It includes a few hundred binary star observations. These are listed in the Double Star Library available on the WEB at ad. usno.navy.milwdsdsl.html, although mixed with many other different observations. For the simple-minded statistics shown in the following, we rely only on the CHARM entries. In the case of multiple systems, we consider only the first two components.

Fig. 3 shows the range of separations for the binary detections reported in CHARM. It should be noted that while interferometric measurements generally report the true separation and position angle of a system, occultation measurements generally report only the quantities projected along the direction of the lunar motion (although there are exceptions to both of these rules). Nevertheless, the figure clearly shows that both techniques are capable of resolving sources with just 1-2 mas. Apart from the different statistics, one can see that LO frequency of detection seem to peak at about 100 mas and decrease for larger separations, while for LBI the peak is around 10 mas. This is just a bias due to the fact that LBI observations are carried out on preselected targets (such as known spectroscopic binaries), while LO are usually observed for field stars. 


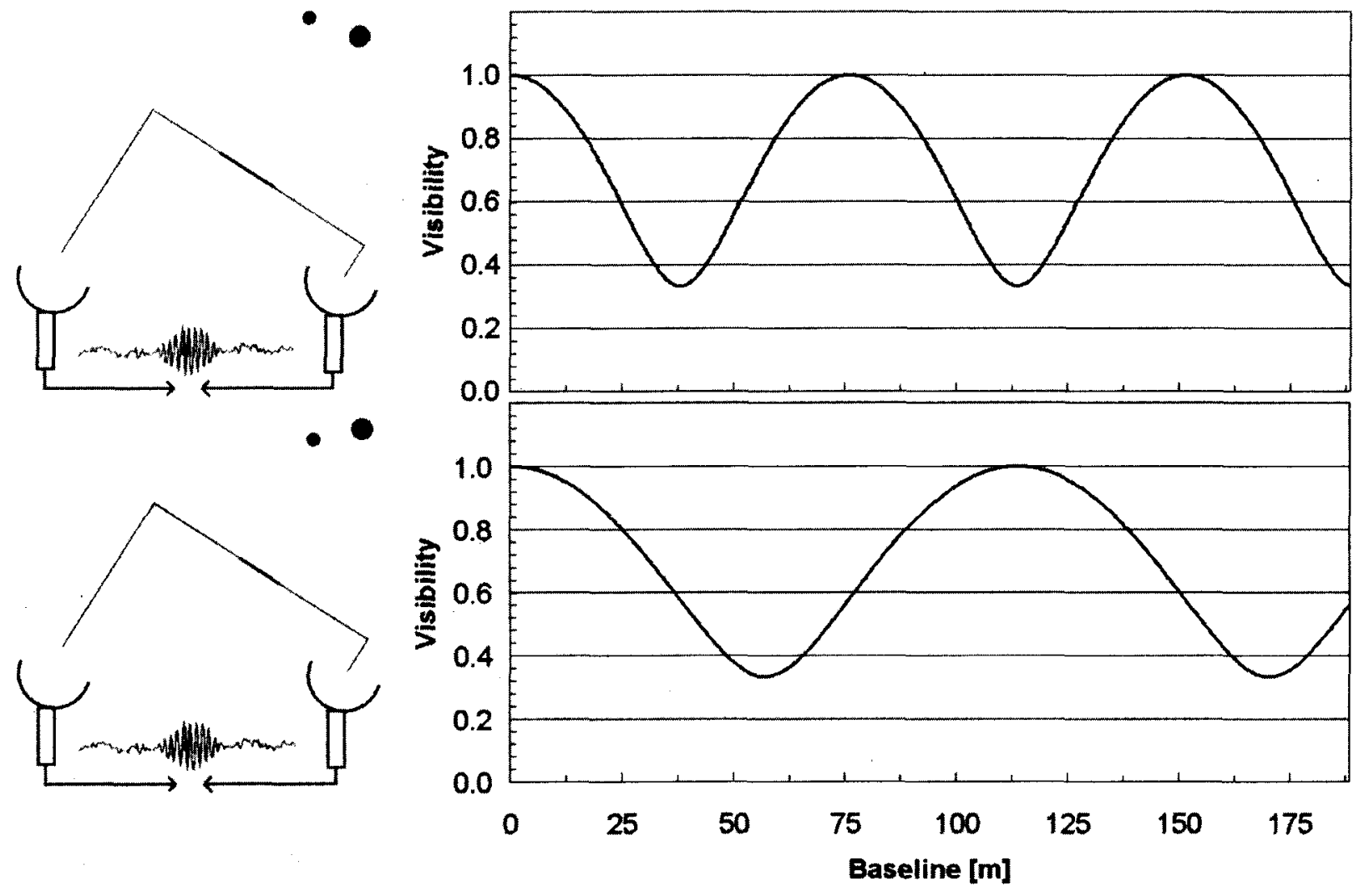

Fig. 2. Schematic representation of a long-baseline interferometry observation of a binary star, and the changes observed. in the visibility curve for two different projected separations.

Fig. 4 shows the range of brightness ratios for the same sample. It should be noted that there is a broad range of wavelengths covered in these entries, from the blue part of the visible spectrum to the near and thermal infrared. One can appreciate also in this case some difference between the two techniques, with LO having a rather broad distribution of brightness ratios and LBI apparently peaking between 2 and 5 . A combination of several factors is at play here, which cannot be easily disentangled in this approximate representation: dynamic range, but also magnitude of the primary, wavelength, angular separation. One could note that the statistics should naturally increase towards higher values of the brightness ratio, and that on the whole LO seem to be more sensitive for fainter companions than LBI.

Finally, Fig. 5 combines the data from the two previous figures, illustrating the overall sensitivity of the two techniques.

\section{CONCLUDING REMARKS}

Now and in the near future, long-baseline interferometry is the technique of choice for the study of close binary systems, to derive accurate orbital

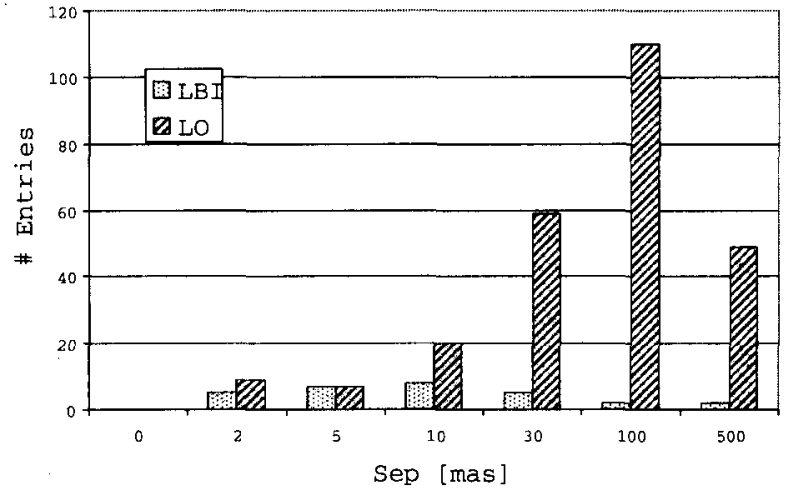

Fig. 3. Statistics of the separations for the entries on binary and multiple systems present in the CHARM catalogue. See text for details.

motions and for the direct detection of low-mass companions. About a dozen major facilities around the world provide baselines of up to several hundred meters, combining telescopes up to the $8-10 \mathrm{~m}$ class. Among the most powerful ones are the Keck Interferometer in the northern hemisphere $(2 \times 10 \mathrm{~m}$ mirrors on a fixed $85 \mathrm{~m}$ baseline), and the ESO 


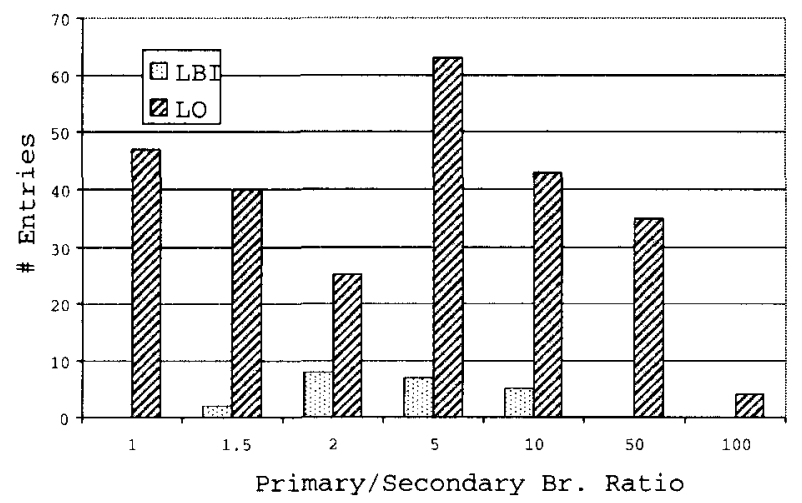

Fig. 4. Statistics of the primary-to-secondary brightness ratio for the entries on binary and multiple systems present in the CHARM catalogue. See text for details.

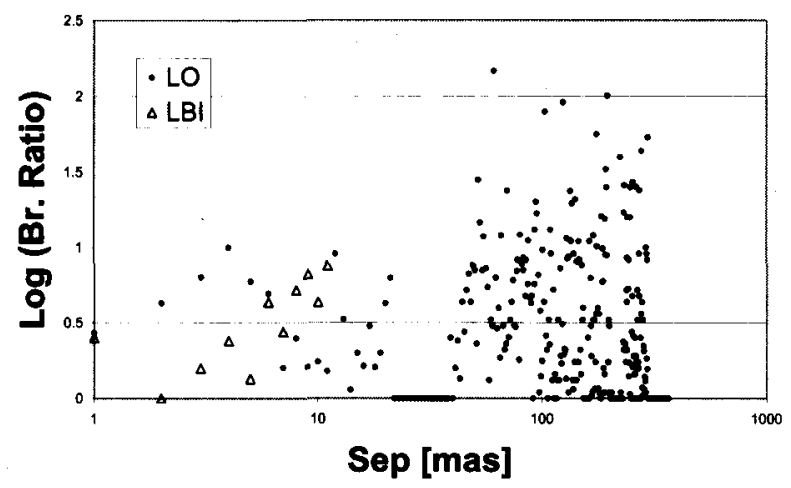

Fig. 5. Combination of the data from Figs. 3-4, showing the sensitivity limits of the LO and LBI techniques. See text for details.

VLT Interferometer in the southern hemisphere, with $4 \times 8 \mathrm{~m}$ and $4 \times 1.8 \mathrm{~m}$ telescopes (these latter movable over 30 stations), with baselines up to $200 \mathrm{~m}$. Several other facilities also offer interesting combinations of multiple telescopes and very long baselines, and we regret not being able to mention them all here (see for example Ridgway \& Roddier 2000).
Angular resolutions at the milliarcsecond level are routinely achieved, with sensitivity of about $\mathrm{K} \approx 8 \mathrm{mag}$. This will be soon expanded at the VLTI to $12 \mathrm{mag}$ with adaptive optics on the large telescopes and $\approx 19 \mathrm{mag}$ with the PRIMA facility (Paresce et al. 2003). This latter will permit narrowangle astrometry at the $10 \mu$ arcsec level (Paresce et al. 2003).

Lunar occultations have reached the peak of their application in the 80's and 90's. Since then, their use has declined in favor of long-baseline interferometry, mainly as a consequence of the limited range of application of the technique. However, it should be stressed here that although limited in the choice and repeatability of observation, lunar occultation are very cheap to implement and do not necessarily need large telescopes. Therefore, they should be an interesting option for many medium-sized observatories, permitting to achieve milliarcsecond resolution with 1-2 m-class telescopes equipped with relatively simple fast photometers.

Due to their intrinsic random nature, lunar occultations are the technique of choice for surveys of binarity among field stars. After observing several hundred occultations, Richichi et al. (2000) reckon that about $14 \%$ of field stars are generally revealed to be new binary systems.

\section{REFERENCES}

Evans, D. S., McWilliam, A., Sandmann, W. H., \& Frueh, M. 1986, AJ, 92, 1210

Hummel, C. A. 2004, this volume

Paresce, F., Delplancke, F., Derie, F., Glindemann, A., Richichi, A., \& Tarenghi, M. 2003, SPIE, 4838, 486

Richichi, A., Leinert, Ch., Jameson, R., \& Zinnecker, H. 1994, A\&A, 287, 145

Richichi, A., \& Percheron, I. 2002, A\&A, 386, 492

Richichi, A., Ragland, S., Calamai, G., Richter, S., \& Stecklum, B. 2000, A\&A, 361, 594

Ridgway, S.T., \& Roddier, F. 2000, SPIE, 4006, 940

\section{DISCUSSION}

Horch - I am curious about the near infrared instrument at VLTI that, as you mentioned, will become available later this year. What will be its limiting magnitude?

Richichi - The Amber instrument is expected to reach a limiting magnitude for point sources of about $\mathrm{K}=12$ and eventually $\mathrm{K}=19$ with the dual-feed facility.

A. Richichi: European Southern Observatory, Karl-Schwarzschildstr. 2, D-85748 Garching b.M., Germany (arichich@eso.org). 